\title{
Working memory and executive function: The influence of content and load on the control of attention
}

\author{
ROBERT HESTER and HUGH GARAVAN \\ Trinity College, Dublin, Ireland
}

\begin{abstract}
In a series of three experiments, increasing working memory (WM) load was demonstrated to reduce the executive control of attention, measured via task-switching and inhibitory control paradigms. Uniquely, our paradigms allowed comparison of the ability to exert executive control when the stimulus was either part of the currently rehearsed memory set or an unrelated distractor item. The results demonstrated a content-specific effect-insofar as switching attention away from, or exerting inhibitory control over, items currently held in WM was especially difficult-compounded by increasing WM load. This finding supports the attentional control theory that active maintenance of competing task goals is critical to executive function and WM capacity; however, it also suggests that the increased salience provided to the contents of WM through active rehearsal exerts a content-specific influence on attentional control. These findings are discussed in relation to cue-induced ruminations, where active rehearsal of evocative information (e.g., negative thoughts in depression or drug-related thoughts in addiction) in WM typically results from environmental cuing. The present study has demonstrated that when information currently maintained in WM is reencountered, it is harder to exert executive control over it. The difficulty with suppressing the processing of these stimuli presumably reinforces the maintenance of these items in WM, due to the greater level of attention they are afforded, and may help to explain how the cue-induced craving/rumination cycle is perpetuated.
\end{abstract}

The ability to keep relevant information in mind is considered a crucial aspect of cognitive function, influencing performance across a range of other areas. Working memory (WM) has been described as the "desktop of the brain" (Logie, 1999), in an attempt to encapsulate the on-line, multitask processing and temporary storage system first outlined by Baddeley and Hitch (1974). Of particular interest here is the proposed role for the central executive, considered to be the most complex but least well-understood component of WM (Baddeley, 1996; Baddeley \& Della Sala, 1998). Baddeley argues that the central executive may in fact be a conglomeration of several subcomponents, servicing at least four separate functions. These include the coordination of separate task performances, switching retrieval strategies for tasks (such as in random generation), selectively attending to a particular stimulus while simultaneously inhibiting a separate stimulus, and manipulating information sourced from the temporary stores.

This research was supported by USPHS Grant DA14100 and GCRC Grant M01 RR00058. We are grateful for the assistance of Kevin Murphy, Catherine Fassbender, Andrea Kübler, Marion Mernagh, Alan Jones, Jason Chan, and Clare O'Neill. Correspondence should be addressed to H. Garavan, Department of Psychology, Trinity College, Dublin 2, Ireland (e-mail: hugh.garavan@tcd.ie).

\section{The Interaction Between Central Executive Resources and Working Memory Capacity}

The recent revision of Baddeley's WM model highlighted the role of central executive resources in strategic processing of information held in the temporary stores (Baddeley, 2001). Baddeley (1996) suggested that the level of performance on the digit-span task, which was argued to involve relatively little complex processing, would be determined primarily by storage rather than by executive function. However, he also cautioned (Baddeley, 1996, 2001) that maximal verbal memory span depended on both the phonological loop and central executive, with participants recruiting central executive resources as the digit load increased past capacity: "As the digit load increased, the demands made on the central executive will increase" (Baddeley, 1996, p. 11). One may infer from this research that maintaining high WM loads requires input from strategic executive processes, such as chunking.

Kane, Engle, and colleagues (Engle, Tuholski, Laughlin, \& Conway, 1999; Kane, Bleckley, Conway, \& Engle, 2001; Kane \& Engle, 2003) have proposed that cognitive measures of WM capacity reflect an individual's capacity to maintain information, including task goals, in a highly active state despite interference. They suggest that keeping relevant information highly active and easily accessible reflects an individual's ability to control attention, because "coherent and goal-oriented behavior in interference-rich conditions requires both the active main- 
tenance of relevant information and the blocking or inhibiting of irrelevant information"(Kane et al., 2001, p. 170). Given this theoretical underpinning, Kane et al. (2001) hypothesized that individual differences in performance on tasks such as the Stroop task and antisaccade task, which are known to be sensitive to inhibitory deficits, might be correlated with an individual's WM capacity. Their results supported this assumption, showing that low-span participants had significantly longer mean target identification times for the antisaccade task and higher interference scores for the Stroop task, than their high-span counterparts, in the absence of any difference between the groups on the comparable control conditions. Both of these results have been taken to support the hypothesis that WM performance represents the capacity to control attention. Therefore, individuals with a high WM span may not necessarily have a greater store of information; rather, they are able to maximize retention of information through the suppression of irrelevant stimuli or responses.

Support for the relationship between the active processing required for WM and inhibitory control is demonstrated in studies such as Roberts, Hager, \& Heron (1994), who first demonstrated that WM and inhibition performance interacted in a behaviorally meaningful way. They used the antisaccade task in combination with a secondary task that varied the level of memory load. They found that the mental arithmetic task used to increase WM load significantly impaired inhibitory task performance. A similar study using the suppression of reflexive saccades as its inhibition task found that performance declined as a function of increasing a secondary WM load (Mitchell, Macrae, \& Gilchrist, 2002). The work of May, Hasher, and Lustig, while providing similar evidence, focused instead on the ability to inhibit proactive interference (PI). Their data suggests that the ability to minimize PI contributes significantly to WM span performance, as evidenced by the reduced WM span of older adults who have increased difficulty with PI, an effect that can be ameliorated if PI is minimized in task trials (Hasher, Chung, May, \& Foong, 2002; Lustig, May, \& Hasher, 2001).

Another example of the relationship between WM and response selection includes the finding that selective visual attention can be influenced by WM load (de Fockert, Rees, Frith, \& Lavie, 2001). This study combined a WM paradigm with a selective visual attention task that asked participants to classify famous written names, such as those of pop stars or politicians, while ignoring either congruent (same name and face) or incongruent distractor faces. This task was performed during the maintenance period of the WM task and was measured using reaction time (RT). The authors identified a significant interaction between the distractor condition and WM load and argued that it was an indication of greater distractor face processing in the selective attention task under high WM loads. Neuroimaging of participants was also undertaken for this task, which indicated that imposing a high WM load increased prefrontal activation (in BA areas 44, 6, and 4). Furthermore, areas previously implicated in face processing - fusiform gyrus, right inferior occipital lobe, and left lingual gyrus - all showed greater activation under a high WM load. de Fockert et al. (2001) argued that the neuroimaging findings supported their assumption that greater WM loads had resulted in increased processing of the distractor faces, which had, in turn, resulted in slower classification times due to the presence of incongruence between faces and names in the selective attention task.

The theoretical models of attentional control (Baddeley, 2002; Shallice \& Burgess, 1998) also argue that the central executive has a major role in the switching of attention. Baddeley, Chincotta, and Adlam (2001) demonstrated in a series of dual-task experiments that secondary central executive tasks interfered with task-switching performance. Furthermore, they showed that a secondary phonological loop task was able to interfere with performance, although from the type of experiments administered the effect appeared to result from interfering with maintenance of the switching program. Despite this evidence, some studies have indicated a lack of relation between individual differences in WM and in task switching (Oberauer, Süß, Schulze, Wilhelm, \& Wittmann, 2000; Oberauer, Süß, Wilhelm, \& Wittmann, 2003). However, few experimental tests, such as those used to establish a relationship between inhibitory control and WM, have been used.

There appears to be good evidence of a relationship between the active processing of information in WM and performance of traditional executive functions such as suppression of prepotent responses. Therefore, our aim in the present study is to demonstrate a common link between WM and executive function that includes both the switching and inhibitory control of attention. Moreover, we wished to explore whether increasing WM load would influence executive control of attention in a materialspecific way. For example, if one increases the number of items held in WM, is it particularly hard to suppress one's prepotent response to (or switch one's attention away from) those items held in WM, or does increasing load simply reduce executive efficiency in an indiscriminate way?

In the present study, we designed two separate paradigms: an inhibitory task and a switching task, which integrated both executive and WM load components into a single task. Previous studies have used designs that combined a WM task with an executive task, although the tasks remained quite separate in their execution (e.g., the tasks used antisaccade methodology). Our designs manipulated the requirement to use the contents of WM in order to successfully complete the executive task, thereby increasing the interaction between the two proposed systems. We hypothesized that performance on both the inhibitory and switching tasks would be compromised by increasing WM load.

\section{EXPERIMENT 1}

Experiment 1 presented a primary WM task that included the occasional requirement to switch to a secondary 
decision making task. The primary WM task was a variation on the standard Sternberg paradigm previously used in studies of WM (Rypma, Berger, \& D'Esposito, 2002), where participants were presented with increasing sets of letters of the alphabet to remember. A series of trials then followed that presented a single letter, and the participant decided whether it had been part of the original memory set. Pseudorandomly placed throughout this series of trials were letters presented in a colored type (task-switching trials). The participant was instructed (and trained) that when a letter appeared in color, he or she was not required to answer if it had been part of the memory set; instead, he or she was to perform a secondary task. This requirement was intended to force the participant to switch from the WM task to a secondary decision making task. It was hypothesized that as WM load increased, the participant's RT to the switch trial would increase due to the requirement to actively maintain larger WM loads in conjunction with maintaining task goals.

The secondary task requirements were manipulated to provide two alternate versions. The first of these required participants to make a judgment on the basis of whether the switch stimulus was a vowel or a consonant. The second version, in which the switch stimuli were presented in either red or green type, the participant indicated its color. The use of different secondary tasks examined whether the processing required for the secondary task influenced the effect of WM load on switch trials, given the debate about early and late processing (Johnston \& Heinz, 1978, 1979; Lavie \& Tsal, 1994). Johnston and Heinz, for example, argued that early or "shallow" processing of information during a decision making task, such as might be required for perceptual qualities of color, required less attentional capacity than did tasks requiring later or "deeper" processing, which we argue the vowel/consonant decision represents. The two versions of the task allow examination of whether the attentional demands of the secondary task, in terms of depth of processing, influence the effect of WM load on taskswitching performance, as well as providing an internal replication of a task-switching effect.

The influence of WM contents on switching performance was also examined through manipulation of the items appearing in colored type. Half the switch items were letters from the memory list. Therefore, on $50 \%$ of task-switching trials participants were required to disengage from the primary WM task and switch to the secondary task in response to a stimulus that was being actively maintained in WM. We hypothesized that this active processing would make it more difficult to disengage from the primary WM task for items from the memory list, and consequently the time to correctly answer the secondary decision making task would be slower than task-switching trials presenting distractor-based letters. We also hypothesized that greater processing of irrelevant information would occur as WM load increased, and therefore an interaction between WM load and contents would be observed during task-switching trials.

\section{Method}

Participants. The participants were 20 undergraduates from the Trinity College Department of Psychology, who received course credit for participation. The participants received both versions of the working memory-switch task (WM-ST), 1 week apart, with the order of the tasks counterbalanced across the sample.

Apparatus, Materials, and Procedure. The E-Prime Version 1.0 program presented the task in 50-point Arial font on a Windowsbased Pentium 133 computer with a 17-in. color monitor and collected latency and accuracy data from keyboard responses.

The WM-ST presented primary WM task trials and secondary task-switching trials. The primary WM task was a variation of the Sternberg memory task. The participants were shown a "memory list" of 2, 5, or 8 uppercase letters. The memory list items were presented for $8 \mathrm{sec}$ in the center of the screen and were immediately followed by a rehearsal period of $6 \mathrm{sec}$ that presented a white crosshair on a black background. A series of 40 test trials then followed, each lasting 2,500 msec, including the presentation of a single letter in white type on a black background for $1,750 \mathrm{msec}$, and a blank black screen for the concluding $750 \mathrm{msec}$. The participant was instructed to respond as quickly as possible as to whether the letter displayed was part of the memory list. The participant was trained to press the "1" key for memory list items and the " 2 " key for distractor items. Each response was trained to a separate finger (index and middle fingers on the right hand, in this example); similarly, the secondary judgment task was always performed with the left hand (index and middle fingers). Training was provided prior to experimental trials to help the participants map stimuli to responses, with 160 memory trials and 70 switch trials administered during various practice runs. The 40 experimental trials per run included an equal distribution of memory and distractor trials, with the memory trials presenting a letter from the memory list and distractor trials presenting random letters from the remaining letters of the alphabet. Accuracy and latency measures were recorded.

The memory lists used in the two versions of the WM-ST were not identical, although both utilized vowels and consonants of the English alphabet. The memory lists for the vowel and consonant (V\&C) version of the WM-ST contained an equal distribution of vowels and consonants within a load size, and the switch items also contained an equal distribution of vowels and consonants.

The color version of the secondary WM-ST presented switch trial stimuli in either red or green type. The participants were instructed (and trained) to press the " $R$ " key for red and the " $G$ " key for green as quickly as possible with their left hand, while using their other hand for the memory list/distractor decision. The use of different keys allowed for the differentiation of commission errors in response to the switch trials. The proportion of memory and distractor switch trials appearing in each color was equivalent, both within and between load conditions. As an additional condition, administered at the beginning of the task, a no-load secondary task was instituted, presenting 30 green and 30 red trials in random order for the participants to indicate the color.

The V\&C version of the secondary WM-ST presented switch trial stimuli in red type, with an equal distribution of vowels and consonants. The participant was instructed (and trained) to respond as quickly as possible to red-type stimuli, pressing the "V" key for vowels and the "C" key for consonants. Again, the proportion of memory and distractor switch trials requiring a vowel or consonant response was equalized to avoid confounds from motor dexterity. The additional no-load condition was instituted for the V\&C secondary task.

Switch trials were embedded pseudorandomly within the presentation of memory trials, with no two switch trials ever appearing back to back. Ten switch trials per run were presented, with an equal distribution of letters from the memory list and distractor letters. The stimulus presentation times from the memory task also applied to the switch trials. 
In total, 30 switch trials and 90 memory trials were presented for each memory load size $(2,5$, and 8$)$. The nine experimental runs (three runs at each load size) were presented in the same sequence for each participant, with the order of presentation counterbalanced for memory load requirements. A 30 -sec rest interval was provided between each run. The tasks, including practice, were performed in separate sessions taking approximately $45 \mathrm{~min}$ each, at least 1 week apart.

\section{Results}

Accuracy performance for both memory and switch trials at each memory load size were calculated for both the color and V\&C versions of the WM-ST and are presented in Table 1 . The accuracy performance for memory trials has been averaged across both positive (from the memory list) and negative (distractor) trials.

The results from Table 1 reflect the expected influence of memory load on memory trial performance. A repeated measures analysis of variance (ANOVA) for memory trials, in which memory load was the within-subjects variable, was performed for both the color and $\mathrm{V} \& \mathrm{C}$ versions of the WM-ST. The results indicated that increasing the number of letters in the memory list resulted in poorer performance on memory trials in the color version $[F(2,38)=$ 58.09, $\left.M S_{\mathrm{e}}=14.83, p<.0001\right]$, as well as in the $\mathrm{V} \& \mathrm{C}$ version $\left[F(2,38)=896.81, M S_{\mathrm{e}}=6.65, p<.0001\right]$. The effect of memory load on performance across the two versions of the WM-ST was also analyzed, indicating that performance was significantly better on the color version $\left[F(2,38)=166.39, M S_{\mathrm{e}}=10.41, p<.0001\right]$, with the advantage being seen at the 8 -item load $[t(19)=$ $14.76, p<.0001]$.

Accuracy on switch trials during the $\mathrm{V} \& \mathrm{C}$ version indicated no significant effects of memory load $[F(2,38)=$ $\left.0.28, M S_{\mathrm{e}}=0.43, p>.05\right]$, switch trial type (memory list, distractor item) $\left[F(1,19)=0.00, M S_{\mathrm{e}}=0.00, p>\right.$ $.05]$, or an interaction $\left[F(2,38)=0.78, M S_{\mathrm{e}}=1.22, p>\right.$ $.05]$. Accuracy for the color version switch trials indicated a small but significant improvement in performance with increasing memory load $[F(2,38)=5.29$, $\left.M S_{\mathrm{e}}=2.72, p<.01\right]$, but no effect of switch trial type (memory list, distractor item) $\left[F(1,19)=0.62, M S_{\mathrm{e}}=\right.$

Table 1

Experiment 1: Accuracy Performance, Represented as the Proportion of Trials Correct, for Both Memory and Switch Trials on the Color and V\&C Versions of the WM-ST From Experiment $1(N=20)$

\begin{tabular}{|c|c|c|c|c|c|c|}
\hline \multirow[b]{3}{*}{ Memory Load } & & & \multicolumn{4}{|c|}{ Switch Trials } \\
\hline & \multicolumn{2}{|c|}{$\underline{\text { Memory Trials }}$} & \multicolumn{2}{|c|}{ Memory List } & \multicolumn{2}{|c|}{ Distractor } \\
\hline & $M$ & $S D$ & $M$ & $S D$ & $M$ & $S D$ \\
\hline \multicolumn{7}{|c|}{ Color Version } \\
\hline 2 letters & .97 & .02 & .91 & .06 & .86 & .08 \\
\hline 5 letters & .95 & .02 & .90 & .07 & .93 & .02 \\
\hline 8 letters & .84 & .02 & .88 & .09 & .94 & .08 \\
\hline \multicolumn{7}{|c|}{ V\&C Version } \\
\hline 2 letters & .97 & .02 & .85 & .13 & .88 & .11 \\
\hline 5 letters & .92 & .03 & .89 & .08 & .87 & .13 \\
\hline 8 letters & .62 & .03 & .87 & .12 & .87 & .12 \\
\hline
\end{tabular}

$0.55, p>.05]$. Inspection of the table of means indicated that the WM-related increases in switch trial performance were driven primarily by the improvement in performance for distractor switch trials, an interaction that was statistically significant $\left[F(2,38)=6.69, M S_{\mathrm{e}}=\right.$ $7.16, p<.01]$.

Mean RT for the no-load switch trials in the color task $(M=507 \mathrm{msec})$ was faster than for the $\mathrm{V} \& \mathrm{C}$ equivalent $(M=592 \mathrm{msec})$. The mean RT for responses to correct memory trials and switch trials was calculated for both the color and $\mathrm{V} \& \mathrm{C}$ versions of the $\mathrm{WM}-\mathrm{ST}$ and presented in Figure 1.

A repeated measures ANOVA was used to test the influence of memory load on memory trial RT, indicating the typical Sternberg effect of increasing RT with increased WM load for both the V\&C $[F(2,38)=61.70$, $\left.M S_{\mathrm{e}}=2,731.99, p<.001\right]$ and color $[F(2,38)=183.54$, $\left.M S_{\mathrm{e}}=1,332.41, p<.001\right]$ versions of the WM-ST.

A repeated measures ANOVA was also conducted to compare the two versions of the WM-ST, in which WM load (2, 5, or 8 letters), switch trial condition (memory list, distractor item), and WM-ST condition (color, V\&C) were within-subjects factors. The purpose of this analysis was to test whether the effect of increasing WM load on switch costs was influenced by the nature of the secondary task. The results demonstrated a significant main effect for WM load $\left[F(2,38)=14.48, M S_{\mathrm{e}}=4,218.64\right.$, $p<.001]$, switch trial condition $\left[F(1,19)=26.91, M S_{\mathrm{e}}=\right.$ $2,386.35, p<.001]$, and a significant interaction between WM load and switch trial condition $[F(2,38)=3.89$, $\left.M S_{\mathrm{e}}=1,405.29, p<.001\right]$. The main effect of WM-ST condition was also significant, but the interaction between WM-ST condition and WM load $[F(2,38)=1.34$, $\left.M S_{\mathrm{e}}=4,081.67, p>.05\right], \mathrm{WM}-\mathrm{ST}$ and switch trial condition $\left[F(1,19)=0.85, M S_{\mathrm{e}}=1,260.70, p>.05\right]$, and WM-ST, WM load, and switch trial condition $[F(2,38)=$ $\left.1.00, M S_{\mathrm{e}}=1,683.64, p>.05\right]$ were not significant. This result indicates that the effect of WM load, switch trial condition, and the interaction between load and condition were not significantly different for the two versions of the WM-ST.

Given that the load $\times$ condition interaction was not significantly different for the two versions of the taskand for purposes of brevity - the main effect of WM load on the switch trial types was analyzed after collapsing across the task condition. The mean RT for memory list switches increased significantly with higher WM load $\left[F(2,38)=16.96, M S_{\mathrm{e}}=2,790.00, p<.001\right]$, with pairwise post hoc comparisons (Bonferroni corrected) indicating that RT latencies for loads of 2 and 5, and 2 and 8 were significantly different. The mean RT for distractor list switches also significantly increased with higher $\mathrm{WM}$ load $\left[F(2,38)=6.96, M S_{\mathrm{e}}=2,833.93, p<.001\right]$, with pairwise post hoc comparisons (Bonferroni corrected) indicating that RT latencies for all comparisons were significantly different.

An alternative analysis, considered post hoc, was to examine the switch costs for returning to the primary 


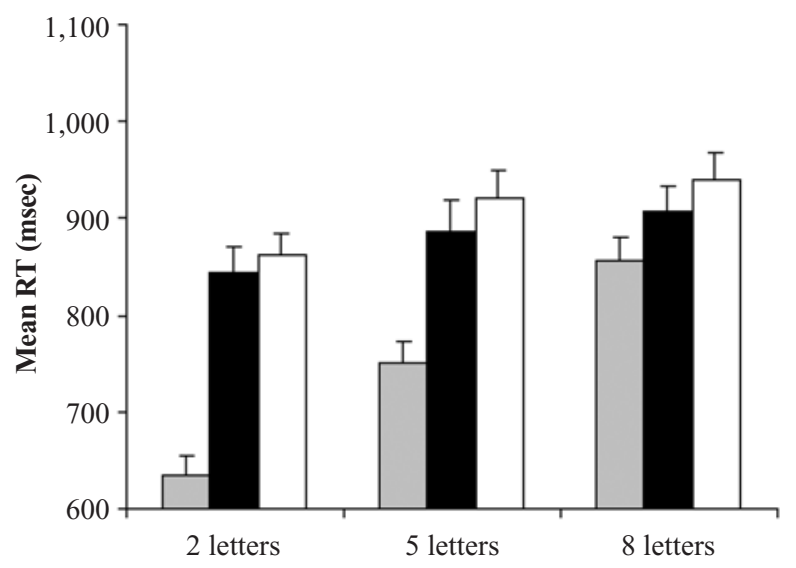

$\square$ Memory Trials $\square$ Distractor Switches $\square$ Memory Switches

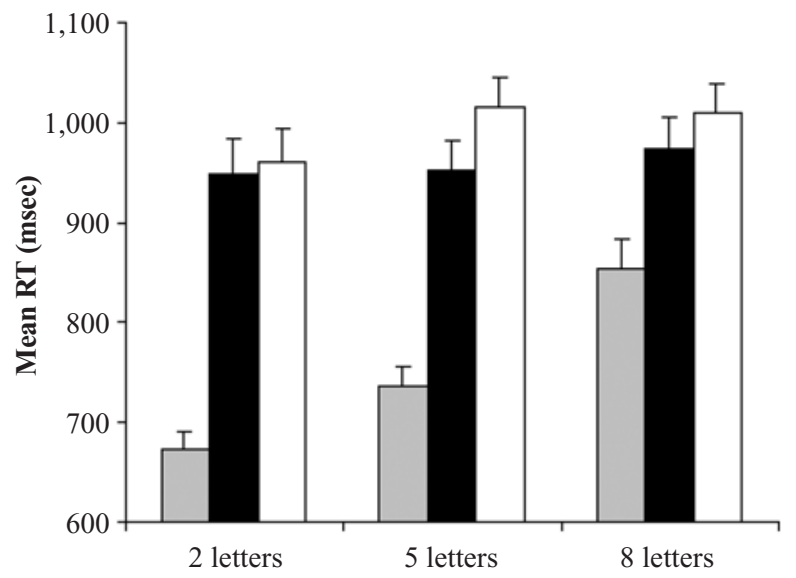

Figure 1. Experiment 1: Mean reaction time for correct memory trials and switch trials on the $\mathrm{V} \& \mathrm{C}$ (top) and color (bottom) versions of the WM-ST. Latencies for switch trials are presented for both letters from the memory list and distractor items. Error bars depict standard errors of the means.

task. Mean RT was calculated for the first (task alternation) and second (task repetition) memory list trials following a switch trial, for each load condition $(2,5$, and $8)$. These statistics were calculated separately for memory and distractor trials, but due to the post hoc nature of the analysis, the task design did not provide equivalent numbers of memory and distractor trials occurring immediately after a switch trial. The switch cost statistic was calculated by subtracting the mean RT for taskrepetition trials from that of the task-alternation trials. The mean switch cost for both memory and distractor items was calculated after collapsing across the color and V\&C versions of the WM-ST and presented in Figure 2.

A repeated measures ANOVA was also conducted to compare the switch costs, in which WM load $(2,5$, or 8 letters) and memory trial condition (memory list, distractor item) were within-subjects factors. The purpose of this analysis was to test whether the effect of increasing WM load on switch costs was influenced by the nature of the item. The analysis demonstrated reliable main effects for WM load $\left[F(2,38)=3.39, M S_{\mathrm{e}}=4,826.26\right.$, $p<.05$ ], with increasing load relating to increased switch cost latencies, but examination of pairwise post hoc comparisons (Bonferroni corrected) indicated that RT latencies for loads of 2 and 8 , but not 5 and 8 , were significantly different. The main effect for memory trial condition was also significant $\left[F(1,19)=13.21, M S_{\mathrm{e}}=3,451.48\right.$, $p \leq .001]$, where switch costs for items that had been part of the memory list were significantly greater than for distractor items. The interaction between WM load and memory trial condition was not significant $[F(2,38)=$ $\left.0.52, M S_{\mathrm{e}}=2,116.48, p>.05\right]$.

\section{Discussion}

The results of Experiment 1 indicate that as the number of items held in WM increased, the speed with which participants were able to switch attention to a secondary decision making task slowed. The manipulation of working memory appeared effective, with response times and accuracy for memory items demonstrating monotonic increases and decreases, respectively. Regardless of the secondary task, holding an increasing WM load on line significantly decreases the speed with which participants can exert executive control. The switch trials during the WM-ST produced longer latencies as a function of the concurrent WM load being rehearsed by participants for the Sternberg-type memory trials. The main effect for WM load was exhibited for both types of switch trials (memory and distractor). This finding is consistent with studies such as Baddeley et al. (2001), which demonstrated the relationship between holding a WM load on line and greater switching latencies, as well as the findings of Kane et al. (2001; Kane \& Engle 2003), who demonstrated a relationship between an individual's working memory capacity and response latencies to incongruent stimuli.

The results of Experiment 1 also appear to extend previous findings by suggesting that the contents of WM have the capacity to directly influence the control of attention. We had hypothesized that the requirement to maintain items in WM would negatively influence the switching of attention, and, furthermore, that when an item held in WM was also the stimulus prompting the switching of attention, the switching cost would increase. The results of Experiment 1 support this assumption, indicating that the time taken to switch attention and perform a secondary judgment task was significantly higher for stimuli that had also been part of the memory list, when compared with distractor items. As predicted, the largest difference between memory and distractor item switches was seen at the highest WM load. Also in support of this hypothesis was the finding of an interaction between WM load and memory status on switching performance, indicating that as WM load increased from 2 to 8 items, the difference between memory and distractor items' switch times increased.

The secondary post hoc analysis of switch costs, derived by subtracting task-repetition from task-alternation performance, also appeared to support these conclusions. The post hoc nature of this analysis somewhat compromises the reliability of its implications; in particular, the 


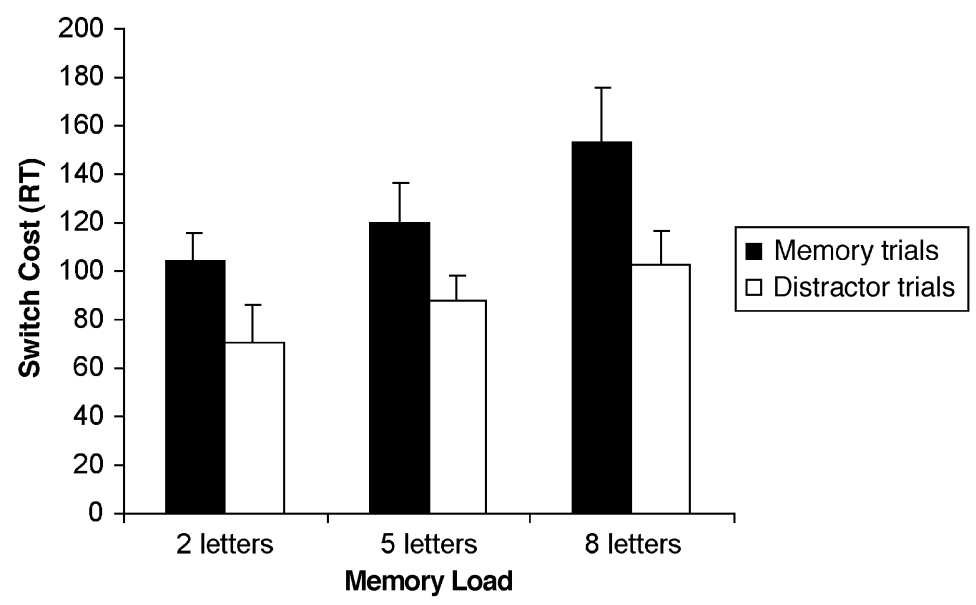

Figure 2. Experiment 1: Mean switch costs (in milliseconds) for memory trials and distractor trials averaged across both the color and $V \& C$ versions of the WM-ST. Error bars depict standard errors of the means.

number of trials used to calculate switch cost statistics varied both within and between load levels. Furthermore, the switch costs derived from this measure may be influenced by the memory content of the switch trial immediately preceding the task-alternation trial. This issue is potentially important, because one hypothesis for the greater switch costs for memory-related items is that participants suppress the processing of items in WM in order to perform the secondary judgment task. Consequently, when returning to the primary task, the previous suppression of the WM items may make them particularly difficult to respond to. Future designs would need to examine, with equivalent amounts of task-alternation trials, what relationship, if any, exists between the memory status of the item prior to and during the task alternation.

One potentially interesting factor arising from Experiment 1 was the impact of secondary task processing on the memory load effect. The present study did not provide support for the secondary task level of processing to influence the effect of WM load and content. The results of the analysis suggested that irrespective of the secondary task performed, the influence of WM load and content remained consistent. One potential confound for this analysis was the poorer WM performance in the $\mathrm{V} \& \mathrm{C}$ version of the WM-ST. It would appear that making a vowel/consonant judgment influences the performance of WM at higher load levels, which is consistent with the hypothesis that secondary tasks requiring higher levels of processing tap into the resources required to maintain high WM loads. Unfortunately, this confounds assessment of the impact of load on switching performance, but it is an interesting finding in its own right because the two tasks were not completed simultaneously.

\section{EXPERIMENT 2}

To examine further the influence of WM load and content on the control of attention, we attempted to general- ize these effects to a different type of executive function: inhibitory control. As indicated in the introduction, increasing WM load has consistently been demonstrated to negatively influence the performance of inhibitory control. However, these previous experiments utilized dual tasks that combined distinct inhibitory and WM tasks, whereas the contents of WM were not required for successful inhibitory control.

The aim of Experiment 2 was to administer a paradigm that would require WM contents to be utilized in order to exert inhibitory control. The design of the task utilized a go/no-go paradigm, which is widely used as a measure of response inhibition in both experimental and clinical studies (Garavan, Ross, Murphy, Roche, \& Stein, 2002; Laurens, Ngan, Bates, Kiehl, \& Liddle, 2003). This type of inhibitory task requires participants to overcome a prepotent motor response. For example, in the present task $91 \%$ of trials presented participants with items that required a buttonpress response, indicating that the item was not part of the WM load presently being rehearsed. This level of prepotency to respond has been argued in previous studies to represent a challenge to inhibitory control (Manly, Robertson, Galloway, \& Hawkins, 1999; Robertson, Manly, Andrade, Baddeley, \& Yiend, 1997).

The "lure" or no-go trials, occurring on $9 \%$ of trials, were items presented as part of the working memory load. We hypothesized, as with the previous literature and the results of Experiment 1, that the simultaneous requirement to actively maintain both task goals and load items in WM would result in a decrease in inhibitory task performance as WM load increased.

\section{Method}

Participants. The participants were 19 undergraduates from the Trinity College Department of Psychology, who received course credit for participation.

Apparatus, Materials, and Procedure. The E-Prime software and computer hardware were identical to those in Experiment 1. 
The working memory-inhibitory task (WM-IT) presented primary WM task trials, within which an inhibitory task was embedded. The primary WM task was a variation of the Sternberg memory task. The participant was shown a memory list of 1,3 , or 5 uppercase letters. The memory list was presented for $6 \mathrm{sec}$ and was immediately followed by a rehearsal period of $6 \mathrm{sec}$ that presented a white crosshair on a black background. A series of 60 test trials then followed, each lasting $1,500 \mathrm{msec}$, including the presentation of a single letter in white type on a black background for 1,000 msec, and a blank screen for the concluding $500 \mathrm{msec}$. The participant was instructed to respond as quickly as possible and to indicate whether or not the letter was part of the memory list. The participant was trained to press the "1" key on the keyboard for letters that were not part of the memory list (distractor items) and withhold his or her response for items that were part of the memory list. The 60 trials included 55 distractor trials and 5 memory item lure trials, which were pseudorandomly placed throughout each run. The purpose of the unequal distribution was to create a prepotent response for the distractor items, in that the participant would become accustomed to pressing the " 1 " key for each trial and would be required to suppress the prepotent response in order to successfully withhold for memory items. In this way, the task is very similar to the random version of the sustained attention to response task (SART; Manly et al., 1999; Robertson et al., 1997), which demonstrated that a probability rate of approximately $11 \%$ for lure trials produces a strong prepotency.

Following the final trial of a run, the participants were asked to recall the letters of the current memory list by typing them on the keyboard. No time limit was imposed for this recall period, and letters could be typed in any order.

In total, 25 memory lure trials and 275 distractor trials were presented for each memory load size (1,3, and 5). Five runs at each set size were administered, with different memory items used during each run. The experimental runs were presented in the same sequence for each participant, with the order of presentation counterbalanced for memory load requirements. A 30-sec rest interval was provided between each run. The task, including practice, was performed in a single session taking approximately $45 \mathrm{~min}$.

\section{Results}

Accuracy performance for both distractor and lure trials at each memory load were calculated and are presented in Figure 3. The results from Figure 3 reflect the expected influence of memory load on distractor trial performance.
A repeated measures ANOVA, in which memory load was the within-subjects variable, indicated that increasing the number of letters in the memory list resulted in poorer performance on distractor trials $[F(2,36)=7.40$, $\left.M S_{\mathrm{e}}=11.45, p<.01\right]$.

The effect of memory load on inhibitory performance within the WM-ST was also analyzed using a repeated measures ANOVA of lure trial performance, indicating that performance was significantly poorer with increasing WM loads $\left[F(2,36)=4.42, M S_{\mathrm{e}}=4.48, p<.05\right]$, with pairwise comparisons indicating that the difference was between a load of 1 and 5 items $[t(18)=3.21, p<.01]$.

Previous studies using inhibitory paradigms such as the antisaccade task (Nieuwenhuis, Ridderinkhof, Blom, Band, \& Kok, 2001), flanker task (Ullsperger \& von Cramon, 2001), and the go/no-go paradigm (Coles, Scheffers, \& Fournier, 1995; Menon, Adleman, White, Glover, \& Reiss, 2001) have identified faster RTs for no-go errors when compared with the mean distractor (go) RT. The RT data were analyzed using a repeated measures ANOVA, indicating that RT for commission errors (Load 1: $246 \mathrm{msec}$; Load 3: $318 \mathrm{msec}$; Load 5: $416 \mathrm{msec}$ ) was significantly faster than distractor RT (Load 1: $349 \mathrm{msec}$; Load 3: $422 \mathrm{msec}$; Load 5: $502 \mathrm{msec})$ for all load levels $[F(1,17)=$ $\left.11.08, M S_{\mathrm{e}}=23,225.73, p<.001\right]$.

The WM recall task indicated that only 1 participant failed to recall all memory lists accurately, and this person incorrectly recalled only one letter from a single list. Apart from this one recall trial, all participants recalled the lists correctly and in their serial presentation order. Participants were not requested to remember or recall the serial order of memory items.

\section{Discussion}

The results of Experiment 2 demonstrate that increasing WM load diminished the ability to exert inhibitory control over a prepotent motor response when the items that required inhibitory control had been rehearsed in WM. The manipulation of WM load appeared effective,

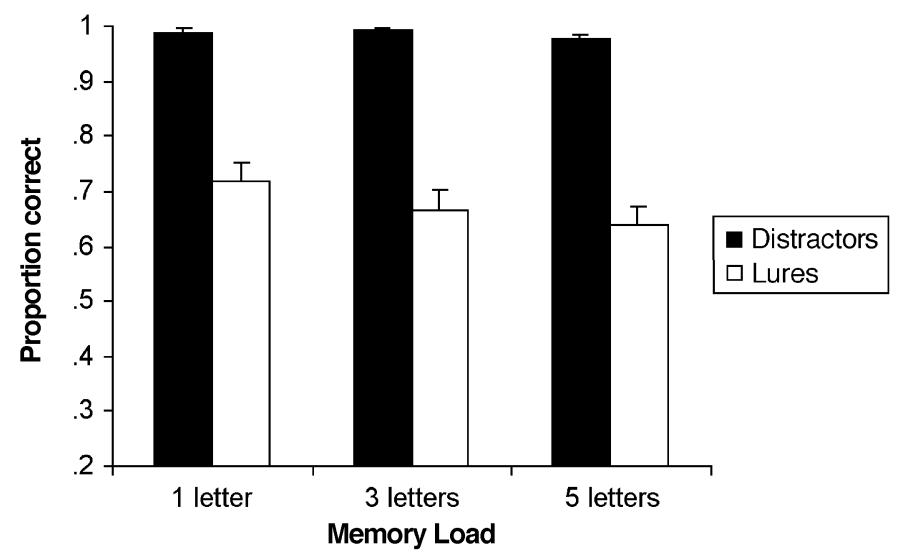

Figure 3. Experiment 2: Accuracy performance, represented as the proportion of correct responses, for both distractor and lure trials on the WM-IT $(N=19)$. 
since response times increased with WM load. Interestingly, participants also recalled memory lists in their presented serial order, suggesting that active maintenance of the items had occurred throughout the task. The finding that increasing WM load negatively influences inhibitory control is consistent with a number of studies demonstrating a relationship between inhibitory control and WM load (de Fockert et al., 2001; Kane et al., 2001; Mitchell et al., 2002; Roberts et al., 1994), while extending the findings of these studies through the use of a paradigm that integrated both the WM and inhibitory components in a single task.

The design of the task attempted to create a prepotency for the primary motor response. Evidence for this can be found in the performance of participants at the 1 -item load level, where they failed approximately $30 \%$ of no-go trials, which is significantly higher than would be expected for a 1-item WM recognition task (Rypma et al., 2002) but is commensurate with other go/no-go tasks where 1 item held in memory must be inhibited (Garavan et al., 2002; Kiehl, Liddle, \& Hopfinger, 2000). Also, mean RT for failed no-go trials was significantly faster than that for correct go trials, which has been previously demonstrated (Coles et al., 1995; Menon et al., 2001), with those authors arguing that errors did not randomly arise - rather, that they are the result of responding due to prepotency before the content of the trial has been fully processed. Similarly, failure to correctly inhibit the prepotent motor response cannot be easily attributed to memory failure. All participants were able to recall each of the memory lists correctly and in serial presentation order, which further suggests that memory lists were actively and serially rehearsed during the task.

In previous designs, participants might reduce the active maintenance of WM items in order to improve their performance on the inhibitory task. Seemingly, this strategy applied to the present task would not improve performance because the contents of WM are required in order to exert inhibitory control. We argue that diminishing inhibitory control with increasing WM load resulted from a simultaneous performance requirement. When a lure item is presented, participants must first cross-reference the item against the list they are rehearsing in WM before they can decide whether to withhold their response. Participants undertook this process for each trial, but for lure trials, the checking of an item currently under rehearsal in WM coincided with the requirement to suppress the prepotent response, a job demanding considerable executive resources (Watanabe et al., 2002).

One limitation of the WM-IT paradigm was the inability to consider the effect of WM content, rather than load, on inhibitory control. In the switching task of Experiment 1 , both load and content could be seen to influence control of attention. The explanation offered for the effect of content was that information held in WM had greater salience, due to its constant rehearsal and, as such, performing the secondary decision making task required suppression of the items actively rehearsed in WM and the prepotent response that went with them. We were interested to see whether the effect of WM content could be demonstrated using the inhibitory control format.

In order to examine this question, we conducted Experiment 3 , testing a different version of the WM-IT. The repeat WM-IT adapted the original to include a second type of inhibitory response where participants were asked to also withhold their response to any stimulus repeated on 2 consecutive trials. Two versions of this task with differing working memory demands were administered. The first version used memory lists of 1,3 , and 5 items as an internal replication of Experiment 2, and the second version, with lists of 2, 5, and 8 items, matched the memory demands of Experiment 1 and the supraspan loads of other studies in the literature.

\section{EXPERIMENT 3}

The repeat WM-IT introduced an additional inhibitory requirement that remained constant throughout the task, irrespective of the number of WM items that also required participants to withhold their response. We assumed that this additional rule also required storage in WM, but this particular requirement would be constant across conditions. The design provided an equal distribution of memoryand distractor-based lures throughout the individual tasks, allowing comparison of the effect of increasing WM load on both types of inhibitory control.

Given the findings of Experiment 2, we hypothesized that increasing WM load would decrease inhibitory performance for memory-related lures. We also hypothesized on the basis of findings from other authors using inhibitory paradigms and our own task-switching paradigm (in Experiment 1) that increasing WM load would have a negative influence of inhibition of distractor-based lures.

\section{Method}

Participants. The participants for the $1 / 3 / 5(n=18)$ and $2 / 5 / 8$ ( $n=16$ ) versions of the repeat WM-IT were two separate samples of undergraduates from the Trinity College Department of Psychology, who received course credit for participation.

Materials and Procedure. The WM-IT introduced in Experiment 2 was adapted to create the repeat version. The primary task remained the same as described in Experiment 2, with participants presented a list of either 1,3 , or 5 letters (memory list) in the $1 / 3 / 5$ version, and 2,5 , or 8 items in the $2 / 5 / 8$ version, which they were required to remember. A series of single letters was then shown, with the participants responding to each of the letters unless it was a member of the memory list, in which case they were to withhold their response. In addition to this task, the participants were instructed to withhold their response if any letter was presented on two consecutive trials.

Following the final trial of a run in either version, the participants were asked to recall the letters of the current memory list by typing them on the keyboard. No time limit was imposed for this recall period, and letters could be typed in any order.

In total, 28 memory-based lures, 28 distractor-based (repeat) lure trials, and 360 distractor trials were presented for each memory load size. The task, including practice, was performed in a single session taking approximately $45 \mathrm{~min}$. The experimental runs were 
presented in the same sequence for each participant, with the order of presentation counterbalanced for memory load requirements. A 30 -sec rest interval was provided between each run.

\section{Results}

Accuracy performance for both memory and repeat lure trials at each memory load were calculated and are presented in Figure 4.

A repeated measures ANOVA was conducted on the results of both versions of the repeat WM-IT, in which WM load (number of letters) and lure type (memory, distractor-based) were within-subjects factors. This analysis tested the influence of increasing WM load on correct inhibition performance for the two types of trials. The analysis for both sets of data revealed the same pattern of performance, with the main effect for lure condition significant for both versions $[1 / 3 / 5: F(1,17)=5.12, p \leq$ $.05 ; 2 / 5 / 8: F(1,15)=4.43, p \leq .05]$, indicating that successful inhibition of memory lures was significantly poorer than the equivalent repeat lure performance. The main effects for the influence of WM load were not significant $[1 / 3 / 5: F(2,34)=2.50, p>.05 ; 2 / 5 / 8: F(2,30)=$ $0.96, p>.05]$. The interaction between WM load and lure condition was, however, significant for both versions $[1 / 3 / 5: F(2,34)=7.71, p<.001 ; 2 / 5 / 8: F(2,30)=$ 9.57, $p<.001]$. The mean performances presented in Figure 4 indicate that increasing memory load decreased memory lure performance $[1 / 3 / 5: F(2,34)=11.69, p<$ $.001 ; 2 / 5 / 8: F(2,30)=14.9, p<.001]$ but did not appear to influence repeat lure performance $[1 / 3 / 5: F(2,34)=$ $3.13, p>.05 ; 2 / 5 / 8: F(2,30)=0.26, p>.05]$.

The mean RT for errors of commission (EoC) on nogo lure trials was also examined for both memory- and distractor-based lures. Analyses were conducted for the high-load version due to the low number of errors for participants in the low-load version and indicated that RT was significantly faster for both types of failed events when compared with correct go RT $(M=471 \mathrm{msec})$ across all memory loads [memory $\operatorname{EoC}(M=443 \mathrm{msec}) F(1,15)=$ $19.1, p<.01$; repeat EoC $(M=427 \mathrm{msec}) F(1,14)=$ $4.51, p<.05]$.

\section{Discussion}

The results of Experiment 3 indicate that as WM load increased, the ability to exert successful inhibitory control over these items decreased. This result was consistent across two versions of the task with varying WM demands and also supports the same finding from Experiment 2 .

The more unexpected result from Experiment 3 was the effect of increasing memory load on performance of the distractor-based lures (repeat lures). It had been hypothesized, given the findings of previous studies (de Fockert et al., 2001; Kane et al., 2001; Mitchell et al., 2002; Roberts et al., 1994), that increasing WM load would decrease all inhibitory control, including inhibition of distractor-based lures. However, the results of Experiment 3 indicated that increasing WM load did not decrease performance for distractor-based lures. This contradictory finding cannot be explained by saying that WM demands were not sufficient to influence performance, with the results indicating that during the eightitem condition only $22 \%$ of memory lists were accurately recalled at the conclusion of a run (mean number of letters recalled $=6.65$ ), and memory lure performance was around $40 \%$.

Another possible account for this effect was the absence of an interaction between distractor-based lures and the underlying memory task. The repeat lures did not bear any relationship to the memory set. Indeed, participants were instructed that this type of lure would not be from the memory set. Perhaps as a consequence, participants were better able to compartmentalize the two tasks and avoid the type of interference found to influence performance for the memory lures and the switching task used in Experiment 1.
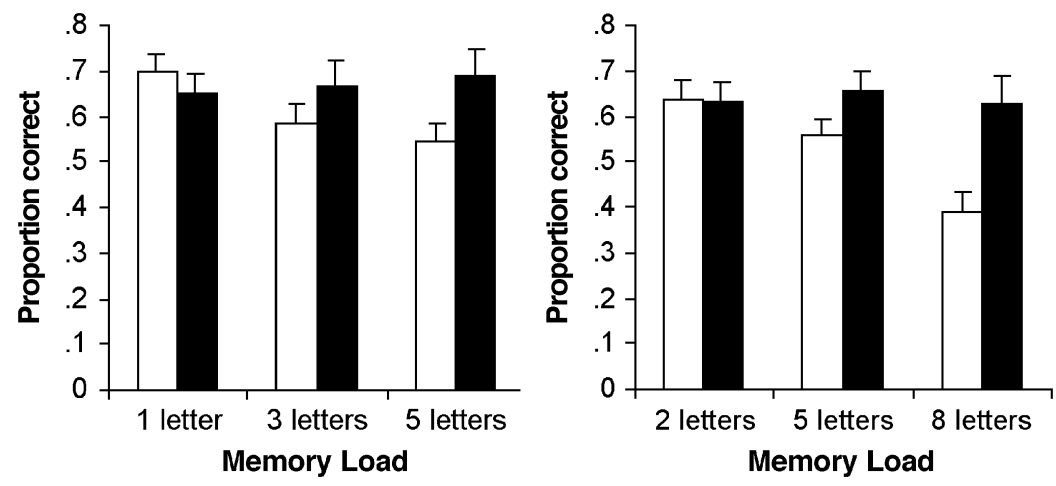

Memory lures

Repeat lures

Figure 4. Experiment 3: Accuracy performance, represented as the proportion of correct responses, for memory and repeat lure trials in the $1 / 3 / 5$ (left graph; $n=18$ ) and 2/5/8 (right graph; $n=16$ ) versions of the repeat WM-IT. 
This finding is also important in that it rules out a "difficulty" explanation for why memory-based inhibitory performance declines with greater WM load. If the introduction of greater WM loads simply made the inhibitory task more difficult, performance for both the memoryand distractor-based lures should have declined. The absence of a decline in distractor-based lure performance provides further support for the memory-based lure decline resulting from a more specific source.

\section{GENERAL DISCUSSION}

In three experiments, we investigated the interaction between WM and executive function, using multiple versions of both the WM-IT and WM-ST. The WM-ST examined the effect of increasing WM load on switching from a primary WM task to a secondary decision making task. The manipulation of interest was whether it would take longer to perform a secondary decision making task on items that were currently being rehearsed for the primary WM task, than for distractor items that bore no relationship to the memory set. Inherent to this task was that participants had to switch from the processing required for the primary task to the decision making processing required for the secondary task. The results from Experiment 1 indicated that large WM loads decreased the speed with which participants could switch from the primary task to the secondary task, and that this effect was particularly apparent for items that had been part of the memory set required for the primary task. The results demonstrated, across two variations in the secondary decision making task, that items currently held in the memory set took significantly longer to switch away from than did distractor items, and that this effect was compounded with increasing WM load.

In Experiments 2 and 3, we demonstrated with an inhibitory paradigm the same pattern of results, with increasing WM load having a deleterious influence on inhibitory control, specifically for those items being held in WM. The task gave participants an increasing number of items to which they were required to inhibit responding. The results suggested that successful inhibitory control over a prepotent response was poorer for items held in WM than for distractor-based related items, and furthermore, that as WM load increased, this effect was compounded.

\section{WM and the Control of Attention}

Kane, Engle, and colleagues (Kane et al., 2001; Kane $\&$ Engle, 2003) argue that the ability to control attention underlies the ability both to inhibit irrelevant processing and to switch between competing tasks. Other models of attention have also grouped these functions; for example, Baddeley $(1996,2001,2002)$ attributes them to the central executive component of his tripartite WM model, and Shallice and Burgess (1998) attribute them to their supervisory attentional system. Among these models is the suggestion that the ability to control attention is in- fluenced by and related to WM. For instance, Kane et al. (2001) argue that controlling attention is, among other things, the ability to maintain a stimulus or goal in the face of interference. WM is important to this process because it contributes to the active maintenance of task goals and information relevant to these goals. In the present study, we aimed to compromise the active maintenance of task goals by requiring more of the active rehearsal in WM to be devoted to maintaining items relevant to the task. Other authors (Miller \& Cohen, 2001; Roberts \& Pennington, 1996) have suggested that when a hierarchy of goal intentions must be maintained, those goals at the bottom of the hierarchy are most likely to be affected by interference from related tasks (i.e., active maintenance of WM loads). The order of goals in the hierarchy is directly influenced by their respective levels of representation, which is in turn determined by the probability that the goal will be enacted. The system of attentional control may become compromised upon the presentation of stimuli that require the enactment of task goals lower in the hierarchy, particularly if the stimuli's salience makes them inherently distracting. Consequently, disorganized behavior may result.

We believe that this hypothesized attentional control system is entirely consistent with the present results. By parametrically increasing the demand for active rehearsal of items in WM, the maintenance of task goals became increasingly compromised. For example, as the number of items requiring a "withhold" response increases in the WM-IT (from 1 to 8 ), the number of task goals competing with the stronger, but ultimately task-irrelevant response (the prepotent response), actually increased. Not surprisingly, the ability to quickly refer to these task goals in order to inhibit a prepotent response became increasingly difficult. Similarly, we predicted that the requirement to switch to the secondary task in the WM-ST would be at the bottom end of the task goal hierarchy, since it was required on only $25 \%$ of the trials. Consequently, as WM demands were increased, switching to the secondary task became increasingly difficult, particularly when distracted by salient stimuli that were being actively rehearsed in WM, just as predicted (Kane \& Engle, 2003; Roberts \& Pennington, 1996). Interestingly, the task goal of withholding in response to the repeat stimuli in the WM-IT was unaffected by increasing WM demands, which while a little surprising, is not inconsistent with this hypothesis, because the likelihood of this task goal being enacted was independent of the WM demands, occurring with the same frequency in each WM load condition.

The memory load effects demonstrated in the present study, when related to Baddeley's model of WM, appear to result from placing greater demands on phonological rehearsal. Other authors have suggested that inner speech within the phonological loop contributes to maintaining the relevant task goal or program, which when burdened with tasks such as articulatory suppression, detracts from, for example, task-switching performance (Baddeley et al., 
2001; Miyake, Emerson, Padilla, \& Ahn, 2004). We believe that the present results offer another example of this effect; burdening the phonological loop with maintaining task-related information detracts from its contribution to task-goal maintenance.

Similarly, other researchers have identified a relationship between WM and the control of attention. This research has suggested that increasing WM load has a deleterious influence on executive function (Baddeley et al., 2001; de Fockert et al., 2001; de Zubicaray, Andrew, Zelaya, Williams, \& Dumanoir, 2000; Mitchell et al., 2002; Roberts et al., 1994). The present study provides further support for this relationship, indicating that performance, either in switching between tasks, or exerting inhibitory control, declines as a function of the WM load being maintained. This relationship was clearly greatest when the WM load was at supraspan levels and required more supraspan-type processing in order to maintain the WM load. For example, performance in the switching task was significantly different when the effect of 2- and 8-item WM loads was compared, whereas loads of 2 and 5 did not demonstrate significant declines. The use of a Sternberg-type paradigm may also have contributed to the small effect sizes, because participants were not required to maintain the serial order of items; such a procedure may have placed a greater demand on WM maintenance at all load levels, but particularly at supraspan levels. The size of these WM loads is clearly a reflection of the sample tested in the present study (college students), but it does suggest that WM loads need to represent supraspan retention for significant negative outcomes to occur for concomitant executive functions.

\section{Controlling Attention Over the Contents of WM}

The present study has seemingly extended the previous findings on WM and the control of attention by demonstrating that both the size and contents of WM have an influence on the control of attention. One interpretation of these findings is that items held in WM have greater salience than do distractor items. As such, they command greater attention when presented to participants than do distractor-based items. This type of assumption is similar to the effect of priming, which has also been shown to capture greater levels of attention (Farah, 1989; Tipper, 2001; Verhaeghen \& Cerella, 2002). If an item has captured a greater level of attention, we might assume that it takes more effort to control the attention afforded to the stimulus. If this assumption is valid, the ability to control attention in the face of the salient WM items would also diminish as a function of increasing WM load, since the present study and others have shown that increasing WM load diminishes these resources (Baddeley et al., 2001; de Fockert et al., 2001; Logan, 1978, 1979; Mitchell et al., 2002; Roberts et al., 1994). The process of rehearsing items in WM maintains this "priming" effect, perpetuating the salience of items held in WM.
Interest in this particular interaction derives from questions concerning the role of WM and executive function in cognitive dimensions of clinical phenomena such as cue-related drug craving. Research suggests the pertinence of cue-related cocaine craving to the activation of a network of cortical regions involved in the engagement of attention, and the subsequent ruminations also involve the frontoparietal network seen in WM rehearsal (Childress et al., 1999; Garavan et al., 2000; Grant et al., 1996; Kilts et al., 2001; Maas et al., 1998). Not surprisingly, drugrelated stimuli were more salient to drug users, and when presented with drug-related items these individuals appeared to activate the cortical areas for rehearsal of items in WM. Other research indicates that individual-specific salient information is maintained in WM with greater accuracy than are distractor items, with this finding being generalized beyond drug users (Franken, Kroon, \& Hendriks, 2000) to studies investigating depression and anxiety (Kulas, Conger, \& Smolin, 2003; Siegle, Steinhauer, Thase, Stenger, \& Carter, 2002).

A cycle appears to occur in which drug-related information has greater salience due to the reinforcing properties of the drug, and when such stimuli are encountered their greater salience makes them easier to remember, and they also appear to trigger rehearsal of the item. The present study has demonstrated that when information, currently maintained in WM, is reencountered, it is harder to exert executive control over it, either by switching away from it, or inhibiting a response to it. Conceivably, salient drug-related stimuli that are cued in the environment start this process of ruminations in WM. As a consequence, when they are reencountered it is more difficult to control the attention directed to them due to the salience of the stimuli and their maintenance in WM. The difficulty with suppression of the processing of these stimuli presumably reinforces the maintenance of these items in WM due to the greater level of attention they are afforded, hence perpetuating the cue-induced craving/rumination cycle. This type of cycle might also be applied to other clinical conditions such as anxiety and depression where ruminations are a symptom (Watkins \& Brown, 2002). It is also apparent that clinical interventions aimed at alleviating cue-induced craving or ruminations may need to eliminate salient items from WM before the cycle of ruminations can be breached (Watkins, Teasdale, \& Williams, 2000).

\section{Conclusion}

In a series of three experiments, increasing WM load was demonstrated to reduce the ability to control attention, measured through both the switching of attention and inhibitory control. Furthermore, the paradigms used in the present study allowed a comparison of the ability to exert executive control when the stimulus was either part of the currently rehearsed memory set or a distractor item. The results demonstrated that as WM loads increased, exerting executive control over the items held in WM became progressively harder than compared with 
equivalent distractor items. These findings are consistent with the idea that WM capacity, especially maintaining supraspan loads, relates directly to the attentional control over functions such as switching of attention and inhibitory control.

\section{REFERENCES}

BAdDEley, A. [D.] (1996). Exploring the central executive. Quarterly Journal of Experimental Psychology, 49A, 5-28.

BADDELEy, A. D. (2001). Is working memory still working? American Psychologist, 56, 851-864.

BADDELEY, A. D. (2002). Fractionating the central executive. In D. T. Stuss \& R. T. Knight (Eds.), Principles of frontal lobe function (pp. 246-260). New York: Oxford University Press.

Baddeley, A. [D.], Chincotta, D., \& Adlam, A. (2001). Working memory and the control of action: Evidence from task switching. Journal of Experimental Psychology: General, 130, 641-657.

BaDdeley, A. [D.], \& Della SAla, S. (1998). Working memory and executive control. In A. C. Roberts, T. W. Robbins, \& L. Weiskrantz (Eds.), The prefrontal cortex: Executive and cognitive functions (pp. 9-21). New York: Oxford University Press.

BADDEley, A. D., \& Hitch, G. J. (1974). Working memory. In G. H. Bower (Ed.), The psychology of learning and motivation (Vol. 8, pp. 47-90). London: Academic Press.

Childress, A. R., Mozley, P. D., McElgin, W., Fitzgerald, J., ReIvich, M., \& O'Brien, C. P. (1999). Limbic activation during cueinduced cocaine craving. American Journal of Psychiatry, 156, 11-18.

Coles, M. G., Scheffers, M. K., \& Fournier, L. (1995). Where did you go wrong? Errors, partial errors, and the nature of human information processing. Acta Psychologica, 90, 129-144.

De Fockert, J. W., Rees, G., Frith, C. D., \& LaVIE, N. (2001). The role of working memory in visual selective attention. Science, 291, 18031806.

de Zubicaray, G. I., Andrew, C., Zelaya, F. O., Williams, S. C., \& DumanoIR, C. (2000). Motor response suppression and the prepotent tendency to respond: A parametric fMRI study. Neuropsychologia, 38, 1280-1291.

Engle, R. W., Tuholski, S. W., Laughlin, J. E., \& Conway, A. R. A. (1999). Working memory, short-term memory, and general fluid intelligence: A latent-variable approach. Journal of Experimental Psychology: General, 128, 309-331.

FARAH, M. J. (1989). Semantic and perceptual priming: How similar are the underlying mechanisms? Journal of Experimental Psychology: Human Perception \& Performance, 15, 188-194.

FRANKEN, I. H., KROON, L. Y., \& HENDRIKS, V. M. (2000). Influence of individual differences in craving and obsessive cocaine thoughts on attentional processes in cocaine abuse patients. Addictive Behaviors, 25, 99-102.

Garavan, H., Pankiewicz, J., Bloom, A., Cho, J. K., Sperry, L., Ross, T. J., Salmeron, B. J., Risinger, R., Kelley, D., \& Stein, E. A. (2000). Cue-induced cocaine craving: Neuroanatomical specificity for drug users and drug stimuli. American Journal of Psychiatry, 157, 1789-1798.

Garavan, H., Ross, T. J., Murphy, K., Roche, R. A., \& Stein, E. A. (2002). Dissociable executive functions in the dynamic control of behavior: Inhibition, error detection, and correction. NeuroImage, 17, 1820-1829.

Grant, S., London, E. D., Newlin, D. B., Villemagne, V. L., Liu, X., Contoreggi, C., Phillips, R. L., Kimes, A. S., \& Margolin, A. (1996). Activation of memory circuits during cue-elicited cocaine craving. Proceedings of the National Academy of Sciences, 93, 1204012045.

Hasher, L., Chung, C., May, C. P., \& Foong, N. (2002). Age, time of testing, and proactive interference. Canadian Journal of Experimental Psychology, 56, 200-207.

JoHnston, W. A. H., \& HeInZ, S. P. (1978). Flexibility and capacity demands of attention. Journal of Experimental Psychology: General, 107, 420-435.

JoHnston, W. A. H., \& HeInZ, S. P. (1979). Depth of nontarget pro- cessing in an attention task. Journal of Experimental Psychology: Human Perception \& Performance, 5, 168-175.

KANE, M. J., BleCKLEY, M. K., CoNWAY, A. R., \& ENGLe, R. W. (2001). A controlled-attention view of working-memory capacity. Journal of Experimental Psychology: General, 130, 169-183.

KANE, M. J., \& ENGLE, R. W. (2003). Working memory capacity and the control of attention: The contribution of goal neglect, response competition, and task set to Stroop interference. Journal of Experimental Psychology: General, 132, 47-70.

KIEHL, K. A., LIDDLE, P. F., \& HOPFINGER, J. B. (2000). Error processing and the rostral anterior cingulate: An event-related fMRI study. Psychophysiology, 37, 216-223.

Kilts, C. D., Schweitzer, J. B., Quinn, C. K., Gross, R. E., Faber, T. L., Muhammad, F., Ely, T. D., Hoffman, J. M., \& DreXler, K. P. (2001). Neural activity related to drug craving in cocaine addiction. Archives of General Psychiatry, 58, 334-341.

Kulas, J. F., Conger, J. C., \& SMOLIN, J. M. (2003). The effects of emotion on memory: An investigation of attentional bias. Journal of Anxiety Disorders, 17, 103-113.

Laurens, K. R., Ngan, E. T., Bates, A. T., Kiehl, K. A., \& Liddle, P. F. (2003). Rostral anterior cingulate cortex dysfunction during error processing in schizophrenia. Brain, 126, 610-622.

Lavie, N. T., \& Tsal, Y. (1994). Perceptual load as a major determinant of the locus of selection in visual attention. Perception \& Psychophysics, 56, 183-197.

LoGAN, G. D. (1978). Attention in character-classification tasks: Evidence for the automaticity of component stages. Journal of Experimental Psychology: General, 107, 32-63.

LoGAN, G. D. (1979). On the use of a concurrent memory load to measure attention and automaticity. Journal of Experimental Psychology: Human Perception \& Performance, 5, 189-207.

LoGIE, R. (1999). Working memory. The Psychologist, 12, 174-178.

Lustig, C., May, C. P., \& Hasher, L. (2001). Working memory span and the role of proactive interference. Journal of Experimental Psychology: General, 130, 199-207.

MaAs, L. C., LuKas, S. E., Kaufman, M. J., Weiss, R. D., Daniels, S. L., Rogers, V. W., Kukes, T. J., \& Renshaw, P. F. (1998). Functional magnetic resonance imaging of human brain activation during cue-induced cocaine craving. American Journal of Psychiatry, 155, 124-126.

Manly, T., Robertson, I. H., Galloway, M., \& Hawkins, K. (1999). The absent mind: Further investigations of sustained attention to response. Neuropsychologia, 37, 661-670.

Menon, V., Adleman, N. E., White, C. D., Glover, G. H., \& Reiss, A. L. (2001). Error-related brain activation during a go/nogo response inhibition task. Human Brain Mapping, 12, 131-143.

Miller, E., \& CoHEN, J. D. (2001). An integrative theory of prefrontal cortex function. Annual Review of Neuroscience, 24, 167-202.

Mitchell, J. P., Macrae, C. N., \& Gilchrist, I. D. (2002). Working memory and the suppression of reflexive saccades. Journal of $\mathrm{Cog}$ nitive Neuroscience, 14, 95-103.

Miyake, A., Emerson, M. J., Padilla, F., \& Ahn, J.-C. (2004). Inner speech as a retrieval aid for task goals: The effects of cue type and articulatory suppression in the random task cuing paradigm. Acta Psychologica, 115, 123-142.

NiEUWENhuis, S., RidDERINKHof, K. R., Blom, J., BAND, G., \& KoK, A. (2001). Error-related brain potentials are differentially related to awareness of response errors: Evidence from an antisaccade task. Psychophysiology, 38, 752-760.

Oberauer, K., SÜß, H.-M., Schulze, R., Wilhelm, O., \& Wittmann, W. W. (2000). Working memory capacity-facets of a cognitive ability construct. Personality \& Individual Differences, 29, 1017-1045.

Oberauer, K., Süß, H.-M., Wilhelm, O., \& WittmanN, W. W. (2003). The multiple faces of working memory: Storage, processing, supervision, and coordination. Intelligence, 31, 167-193.

Roberts, R. J., Jr., Hager, L. D., \& Heron, C. (1994). Prefrontal cognitive processes: Working memory and inhibition in the antisaccade task. Journal of Experimental Psychology: General, 123, 374-393.

Roberts, R. J., JR., \& PenNington, B. F. (1996). An interactive framework for examining prefrontal cognitive processes. Developmental Neuropsychology, 12, 105-126. 
Robertson, I. H., Manly, T., Andrade, J., Baddeley, B. T., \& Yiend, J. (1997). “Oops!": Performance correlates of everyday attentional failures in traumatic brain injured and normal subjects. Neuropsychologia, 35, 747-758.

RYPMA, B., BERGER, J. S., \& D'EsPosito, M. (2002). The influence of working-memory demand and subject performance on prefrontal cortical activity. Journal of Cognitive Neuroscience, 14, 721-731.

Shallice, T., \& Burgess, P. (1998). The domain of supervisory processes and the temporal organization of behaviour. In A. C. Roberts \& T. W. Robbins (Eds.), The prefrontal cortex: Executive and cognitive functions (pp. 22-35). Oxford: Oxford University Press.

Siegle, G. J., Steinhauer, S. R., Thase, M. E., Stenger, V. A., \& CARTER, C. S. (2002). Can't shake that feeling: Event-related fMRI assessment of sustained amygdala activity in response to emotional information in depressed individuals. Biological Psychiatry, 51, 693707.

TIPPER, S. P. (2001). Does negative priming reflect inhibitory mechanisms? A review and integration of conflicting views. Quarterly Journal of Experimental Psychology, 54A, 321-343.

Ullsperger, M., \& von Cramon, D. Y. (2001). Subprocesses of perfor- mance monitoring: A dissociation of error processing and response competition revealed by event-related fMRI and ERPs. NeuroImage, 14, 1387-1401.

Verhaeghen, P., \& Cerella, J. (2002). Aging, executive control, and attention: A review of meta-analyses. Neuroscience \& Biobehavioral Reviews, 26, 849-857.

Watanabe, J., Sugiura, M., Sato, K., Sato, Y., Maeda, Y., Matsue, Y., Fukuda, H., \& Kawashima, R. (2002). The human prefrontal and parietal association cortices are involved in no-go performances: An event-related fMRI study. NeuroImage, 17, 1207-1216.

WATKIns, E., \& Brown, R. G. (2002). Rumination and executive function in depression: An experimental study. Journal of Neurology, Neurosurgery \& Psychiatry, 72, 400-402.

WatKins, E., Teasdale, J. D., \& Williams, R. M. (2000). Decentering and distraction reduce overgeneral autobiographical memory in depression. Psychological Medicine, 30, 911-920.

(Manuscript received August 19, 2003; revision accepted for publication June 4, 2004.) 